\title{
An Energy-efficient Mobile Routing Algorithm in Wireless Sensor Network
}

\author{
Lin Zhigui ${ }^{1}{ }^{*}$, Li Min ${ }^{1}$, Liu Yingping ${ }^{2}$, Yang Ziyuan ${ }^{3}$ and Zhang Huiqi ${ }^{1}$ \\ 1. School of Electronics and Information Engineering, Tianjin Polytechnic \\ University, Tianjin 300387, China; 2. School of Mechanical Engineering, Tianjin \\ Polytechnic University, Tianjin 300387, China; 3. Laboratory of marine \\ environment observation and monitoring technology of offshore, National Ocean \\ Technology Center, Tianjin 300112, China \\ *.linzhigui@tjpu.edu.cn
}

\begin{abstract}
In order to extend the lifetime of wireless sensor network and avoid network premature invalidation caused by local emergency, this paper proposes an energy-efficient mobile routing algorithm (MREEMRP). The network based on the MREEMRP is divided into several square virtual grids to reduce energy consumption, and the cluster head is selected according to the residual energy of nodes and the weighted sum of coordinate distance between a node and the cluster barycenter. The sink node receives data from the cluster head by controllable moving scheduling strategy, and relay nodes with unlimited energy are introduces to extend the network lifetime, and serve as information transmitting large area. On this basis, the simulation is carried out and the results are compared with the MSEERP algorithm without relay nodes. The analysis shows that the proposed algorithm is superior to the MSEERP algorithm from the aspects of total energy consumption of the network, active nodes number and data amount that the gathering node receives.
\end{abstract}

Keywords : Mobile relays; Clustering routing; Energy-efficient; WSN

\section{Introduction}

For energy constrained characteristics of wireless sensor networks, a mobile node without energy limitation is widely used for transmitting information in order to save the network energy and extend the network lifetime. The mobile node may be an ordinary node or a sink one [1]. If it is an ordinary node, the function of each node is identical and no main coordinator node exists in the network, which brings difficulty to control the mobile node; if it is a sink one, the number of mobile nodes is less and the control is relatively simple, but the effects of the sink nodes are different under different mobile strategies[2].

Moving strategies of the mobile sink can be divided into three categories, including random moving strategy, predictable moving strategy and controllable moving strategy. For the random moving strategy [3], such as TRAIL[4] and TTDD protocol[5], the sink position is selected randomly, and the mobile sink radios its location information frequently which consumes a lot of energy. For the predictable moving strategy, the sink trajectory is predetermined, but its flexibility is poor and the network size is not extendable. However, for the controllable moving strategy[6], the sink movement direction is determined by some parameters of the network, and the mobile sink node can move purposefully to prolong the network lifetime. In the mobile sink network, communication modes of the sink include not only single hop and multi-hop communication, but also active data collection and limited number of hop communication. 
During the process of active data collection ${ }^{[7]}$, the mobile sink can access all the nodes in the network and collect the required data, but the delay of data transmission is longer under this communication mode, such as QCCA protocol[5]. Nevertheless, in limited number of hops[8], the mobile sink radios and receives message within a limited transmission range, and the energy consumption of forwarding path can be effectively reduced, such as TTDD protocol [9].

When wireless sensor network encounters sudden events (such as local fire in forest, local pollution in water and so on), the data may increase suddenly, which will cause sudden region routing problems. It is difficult to solve this kind of problem using routing protocol with mobile sink node. Although the ordinary mobile node can solve this kind of problem, it is difficult to control the mobile node and the routing protocol is complicated. For the static wireless sensor networks, Wang [10] introduced mobile relay nodes in the static network where energy is not restricted, which well solved the routing problem caused by large amount of local information from sudden events. Inspired by Wang's idea, this paper introduces a mobile sink node and a mobile relay node without energy constraints, and an energy-efficient mobile routing algorithm (MREEMRP) is proposed. The algorithm reduces the routing path length and balances the network energy consumption by the mobile sink node, so that the information amount in the local network and high energy consumption by the mobile relay node can be reduced significantly, which extends the network lifetime effectively.

The structure of the paper is as follows: the MREEMRP algorithm is proposed, and central idea of MREEMRP is discussed in Section II. Section III introduces the mobile node, and analyzes moving process of mobile relay and sink node. Section IV describes flow of the MREEMRP algorithm. The MREEMRP and MSEERP algorithm are compared and analyzed in Section V. Finally, Section VI summarizes and concludes.

\section{The MREEMRP Algorithm}

\subsection{Basic Conceptions}

The monitoring area is divided into fixed square virtual grids, and the nodes are assigned to each grid according to their location information. Each grid is viewed as a cluster, and the cluster head is selected by the residual energy of each node and the weighted sum of coordinate distance between a node and the cluster barycenter, which ensures the energy consumption of data transmission in the cluster is least, and avoids the node with less residual energy is selected as cluster head. In each grid, only cluster head keeps active, while other nodes go into sleep state. The sink node radios limited number of hops and uses the controllable moving strategy, namely the sink does not collect the information until it moves to the cluster with highest residual energy.

The network introduces a mobile relay node which can locate its own position and move freely in the network, and thus realizes the broadcast of its position information on other nodes. The mobile relay node has no energy constraints and its wireless transmission capacity is similar to an ordinary node. When the mobile relay node moves near to an ordinary node, it takes the place of the ordinary node to bear all forwarding task, whereas the ordinary node only needs to send its perception data.

\subsection{Grid Mesh}

The MREEMRP divides the network into several square virtual grids ${ }^{[11]}$. Suppose the area of the network is $\mathrm{S}$, the radio communication rang between any two nodes is $\mathrm{R}$ and the virtual grid size is $r$. In order to make the network connected, the virtual grid size should satisfy formula (1).

$$
r^{2}+\left(2 r^{2}\right) \leq R=>\frac{R}{\sqrt{5}}
$$


The network is divided into $n\left(n=S / r^{2}\right)$ numbered grids, and each grid is treated as a cluster. Then, the nodes are assigned to a cluster according to their location information as follows. Assuming the coordinates of a node are $(x, y)$, if $x$ and $y$ satisfy $(r *(i-1) \leq x \leq r * i, r *(j-1) \leq y \leq r * j)$, the node belongs to the cluster $(i, j)$. Figure 1 shows an example of clustering in the MREEMRP.

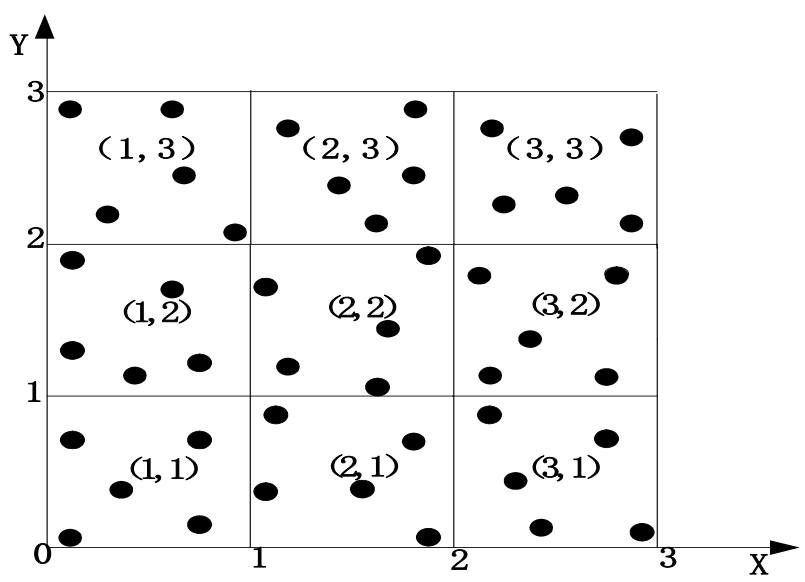

Figure 1. Clustering Diagram

\subsection{Selection of Cluster Head}

After grids are divided, a cluster head should be selected for each grid.

Firstly, areal coordinates of each grid should be calculated. Areal coordinates $\left(\mathrm{x}_{\mathrm{g}}, \mathrm{y}_{\mathrm{g}}\right)$ of a grid satisfy the characteristic of minimizing distance square sum of any node coordinates in the grid, so that they are calculated by coordinates of all nodes in the grid according to the calculation method shown in formula (2).

$$
\left(x_{g}, y_{g}=\min \sum_{i=1}^{N}\left[\left(x-x_{i}\right)^{2}+\left(y-y_{i}\right)^{2}\right]\right)
$$

where the coordinates of the node $i$ in the grid are $\left(\mathrm{x}_{\mathrm{i}}, \mathrm{y}_{\mathrm{i}}\right)$.

By transformation of formula (2), the areal coordinates of each grid is obtained as formula (3) shows.

$$
x_{g}=\frac{1}{2} \sum_{i=1}^{N} x_{i}, y_{g}=\frac{1}{N} \sum_{i=1}^{N} y_{i}
$$

Whether a node can be selected as a cluster head depends on its residual energy and the weighted sum of coordinate distance between a node and the cluster barycenter. Denote the weighted sum of the node $j$ as $f_{\mathrm{j}}$, and then $f_{\mathrm{j}}$ is calculated by formula (4):

$$
f_{j}=\alpha E_{j}+(1-\alpha) / d_{j} \quad \alpha \in(0,1)
$$

where $\alpha$ is weighting coefficient, Ej is node residual energy, and dj is distance of the node $\mathrm{j}$ to the areal coordinates.

Assuming coordinates of the node $j$ are $\left(\mathrm{x}_{\mathrm{j}}, \mathrm{y}_{\mathrm{j}}\right)$, then the distance $d_{j}$ is calculated using formula (5).

$$
d=\sqrt{\left(x_{g}-x_{j}\right)^{2}+\left(y_{g}-y_{j}\right)^{2}}
$$

By comparing the weight of each node in the same cluster, the node with maximum value of $f_{j}$ is selected as the cluster head. Namely, the probability that the node with more residual energy and closer to the areal coordinates is selected as the cluster head is relatively large. Besides, as a weighting coefficient, if a larger $\alpha$ value is chosen, it means that node residual energy is more important, or that the distance between the node and areal coordinates is not so important. An example of clustering in the MSEERRP is shown in Figure 2. 


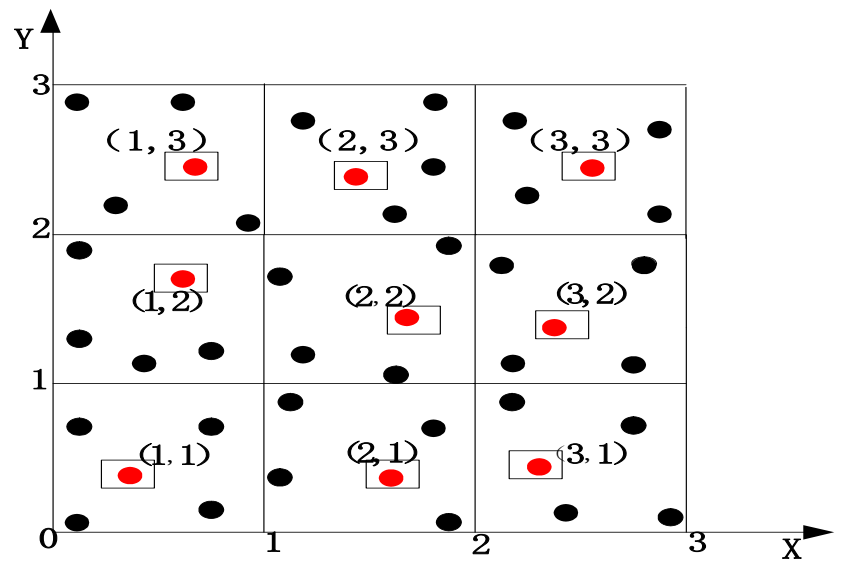

Figure 2. The Result of Selecting Cluster Head

A round of data transmission is that all cluster heads of the network are accessed by mobile sink, and a new cluster head should be re-selected after the round ends to balance energy consumption of the whole network. Supposing the period of every round is $T$, then $\mathrm{T}$ can be calculated using formula (6).

$$
T=\sum_{i=0}^{n-1} \sqrt{\left(x_{i+1}-x_{i}\right)^{2}+\left(y_{i+1}-y_{i}\right)^{2}} / V
$$

where $\mathrm{V}$ is speed of the mobile sink.

\section{Moving Process of Mobile Node}

\subsection{Moving Process of Sink Node}

Moving mode of the sink node combines the characteristics of static mode and movable mode. The sink node receives or sends data in stationary state, but moves by controllable moving strategy and radios message in limited transmission range in mobile state.

Suppose the mobile sink radios a hello packet as shown in Figure 3 in the range of two hops. If a cluster head receives the packet, it calculates average residual energy of the cluster (Ea) according to formula (7):

$$
E \alpha=\sum_{i=1}^{N-1} E_{i} / N
$$

where, $\mathrm{N}$ is the number of nodes in the cluster. Then, the packet accesses the information of its grid number, position and $\mathrm{Ea}$, and returns to the mobile sink. By comparing the average residual energy of each cluster, the sink selects the cluster with the largest average energy. Afterwards, the sink radios message packet containing grid number of the selected cluster head, as shown in Figure 4. When a cluster head receives the packet, grid number of the packet is compared with that of the cluster. If the two numbers are equal, then the cluster gets ready for sending data, or the packet should be discarded. After the mobile sink arrives at destination, the cluster head transmits data to the sink. The mobile sink stays for a period of time, and then radios the hello packet once again. 

Residual Energy; and CHL is Cluster Head Position.)

\section{\begin{tabular}{l|l|l} 
Cluster ID & $\mathrm{T}$ \\
\hline
\end{tabular}}

\section{Figure 4. Message Packet Format}

\subsection{Moving Process of Mobile Relay}

In the network, the route of mobile relay node is a series of concentric circles. The concentric circles take the network center as the center, and expand their radii until reaching the boundary of the network. The mobile relay node stays at each sensor node for a time to replace the data packet forwarding.

During the running of the network, the ordinary node determines the position of mobile relay node. If it has the same position with the mobile relay node, it will send the data to mobile relay node, or the data will be sent to a neighbor node closer to the cluster head node. If the mobile relay node moves to the cluster head node, it will send the data to the mobile sink node instead of the cluster head node. Thus, the mobile relay node can replace other nodes to transmit data. As Figure 5 shows, the mobile relay node replaces node A to transmit data when it arrives at area of the node A, and replaces the cluster head node when it arrives at area of the cluster head to send the collected data to the sink node.

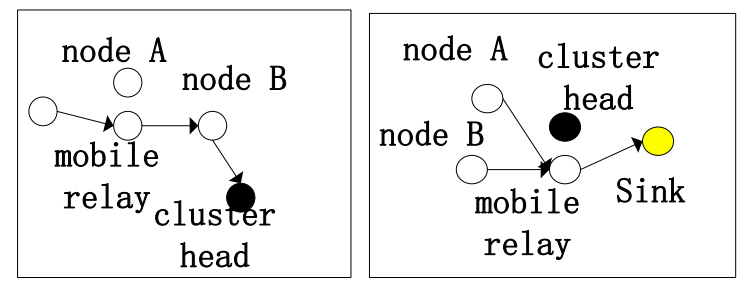

Figure 5. Schematic Diagram of Mobile Relay Node Forwarding

\section{Flow of the MREEMRP}

The MREEMR mainly comprises network clustering, mobile sink moving and mobile relay moving. Each sensor node, before information transmission, needs to judge whether the mobile relay arrives. If it arrives, the transmitted information is forwarded to the mobile relay for further transmission; otherwise the node transmits the data by itself. The flowchart of the MREEMRP is shown in Figure 6. 


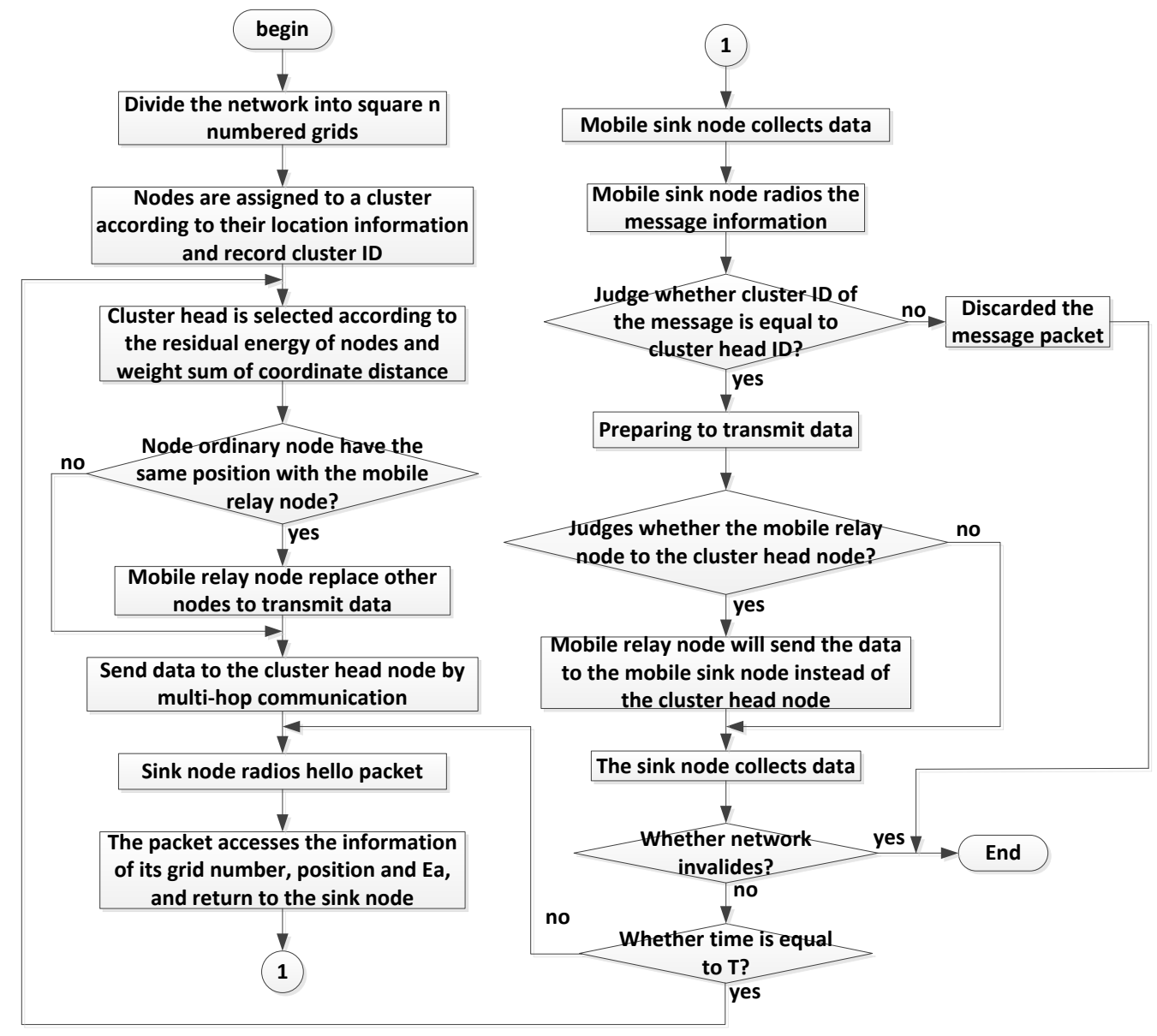

Figure 6 Flowchart of the MREEMRP

\section{Simulation and Analysis}

\subsection{Simulation Environment}

The MSEERP is simulated by the NS2. The network size is set as $100 \mathrm{~m} * 100 \mathrm{~m}$. The number of nodes is set as 100 and the position of nodes is randomly initialized. Other simulation parameters are listed in Table 1.

Table 1. Simulation Parameters

\begin{tabular}{|c|c|c|}
\hline Parameter & $\begin{array}{l}\text { Parameter } \\
\text { value }\end{array}$ & Remarks \\
\hline $\mathrm{S}$ & $100 \mathrm{~m} * 100 \mathrm{~m}$ & Network size \\
\hline $\mathrm{N}$ & 100 & Number of nodes \\
\hline $\begin{array}{l}M A C_{p} \\
\mathrm{E}_{\text {gink }}\end{array}$ & IEEE802.15.4 & MAC Protocol \\
\hline $\begin{array}{c}\mathrm{L}_{\text {sink }} \\
\mathrm{E}_{\text {init }}\end{array}$ & $+\infty$ & $\begin{array}{l}\text { Energy of sink } \\
\text { Primary eneroy }\end{array}$ \\
\hline$\alpha$ & & sensor nodes \\
\hline u. & 0.6 & Weighting coefficient \\
\hline Vs & $5 \mathrm{~m} / \mathrm{s}$ & The moving speed of \\
\hline $\mathrm{Vr}$ & $1 \mathrm{~m} / \mathrm{s}$ & $\begin{array}{l}\text { sink } \\
\text { The moving speed of }\end{array}$ \\
\hline
\end{tabular}




\begin{tabular}{|c|c|c|}
\hline & & mobile relay \\
\hline $\mathrm{R}$ & $50 \mathrm{~m}$ & $\begin{array}{l}\text { Communication } \\
\text { radius of node }\end{array}$ \\
\hline $\mathrm{I}_{\max }$ & $2000 \mathrm{~s}$ & $\begin{array}{l}\text { Maximum time for } \\
\text { simulation each time }\end{array}$ \\
\hline $\mathrm{E}_{\text {elec }}$ & $50 \mathrm{~nJ} / \mathrm{bit}$ & $\begin{array}{l}\text { Energy consumption } \\
\text { of radioing or } \\
\text { receiving } 1 \text { bit }\end{array}$ \\
\hline$\varepsilon_{\mathrm{fs}}$ & $10 \mathrm{~nJ} / \mathrm{bit} / \mathrm{m}^{2}$ & $\begin{array}{l}\text { Energy consumption } \\
\text { parameter for free } \\
\text { space model }\end{array}$ \\
\hline$\varepsilon_{\mathrm{amp}}$ & $\begin{array}{c}0.0013 \mathrm{pJ} / \mathrm{bit} / \\
\mathrm{m}^{4}\end{array}$ & $\begin{array}{c}\text { Energy consumption } \\
\text { parameter for } \\
\text { multi-path fading } \\
\text { model }\end{array}$ \\
\hline
\end{tabular}

\subsection{Comparative Analysis of Simulation Results}

Based on the NS2, the simulation results of the MREEMRP and the proposed MSEERP [12] are compared from the aspects of total energy consumption of the network, active node number and data amount collected by sink.

(1) Total energy consumption of the network: Total energy consumption of the network is shown in Figure 7, where the horizontal axis represents the network running time and the vertical axis represents the network total energy consumption. As Figure 7 shows, when $t$ belongs to $(0,100)$, the consumption energy of the MSEERP is slightly higher than that of the MREEMRP; when $t$ is greater than $100 \mathrm{~s}$, the MREEMRP begins to demonstrate its superiority; when the $t$ is equal to $500 \mathrm{~s}$, energy consumption of the MSEERP is about $144 \mathrm{~J}$ while that of the MREEMRP is about $117 \mathrm{~J}$, that is to say, energy consumption of the MREEMRP decreases by 23\% relative to the MSEERP. To sum up, the MREEMRP presents slower energy consumption, and consumes less energy in the whole process from 0 to $500 \mathrm{~s}$.

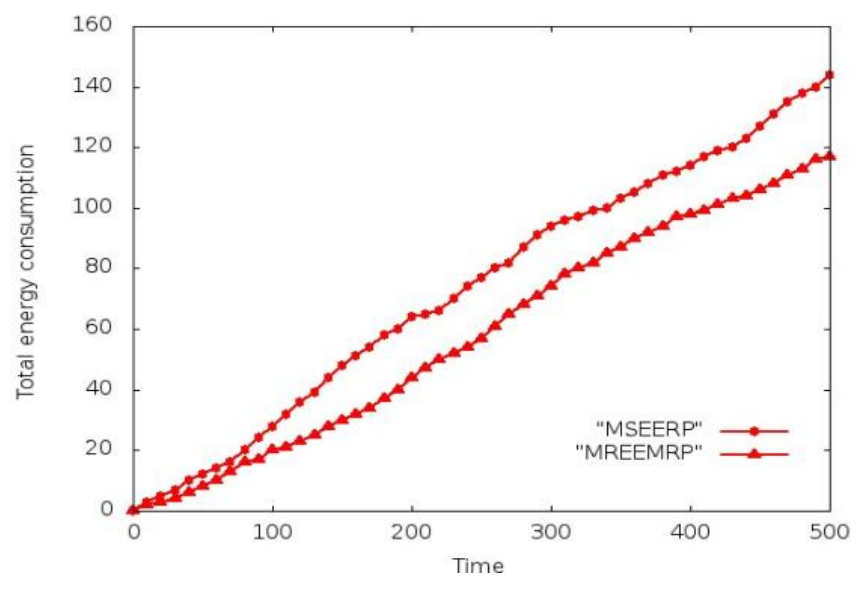

Figure 7. Comparison of the Total Energy Consumption

(2) Active node number: Figure 8 shows the variation trend of active node number, where the horizontal axis represents the network running time and the vertical axis represents the number of active nodes. For the MSEERP, node death appears when $t$ is equal to $1220 \mathrm{~s}$ because of energy depletion; when $t$ is equal to $1490 \mathrm{~s}$, about $50 \%$ nodes 
are dead; when $t$ is equal to1779 $\mathrm{s}$, almost all nodes are dead, and the simulation is killed. Whereas for the MREEMRP, node death appears when $t$ is equal to $2420 \mathrm{~s}$; half of nodes are dead when $t$ is equal $2770 \mathrm{~s}$; and the network lifetime goes to the end when $t$ is equal to 3118 s. Compared with the MSEERP, the time when the first dead node of the MREEMRP delays for $1200 \mathrm{~s}$, and the network lifetime also extends by $75.3 \%$.

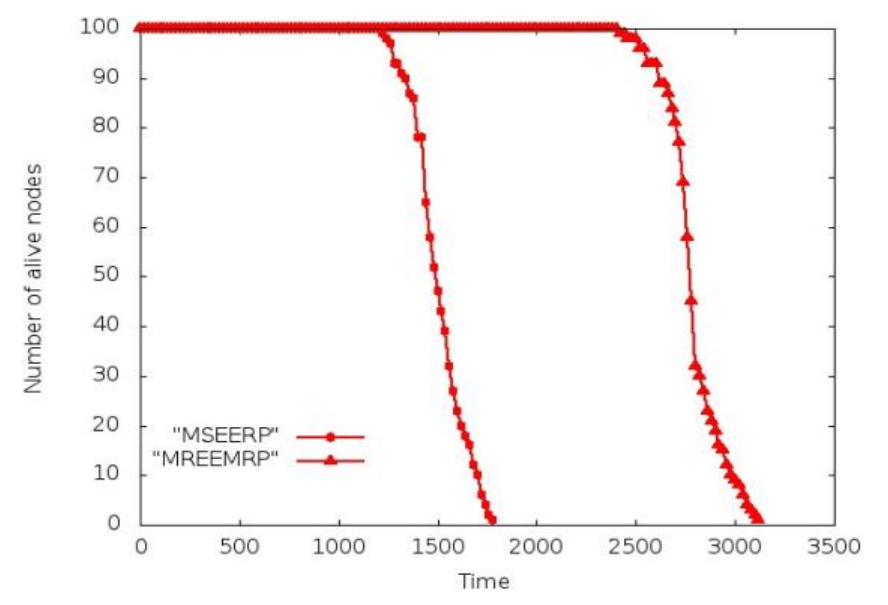

Figure 8. Comparison of the Number of Active Nodes

(3) Data amount received by sink node: The comparison of data packet received by sink node is shown in Figure 9, where the horizontal axis represents the network running time, and the vertical axis represents the number of received packet by the sink node. When $t$ belongs to $(0,100)$, the received packet of the sink nodes by the MSEERP is the same as that by the MREEMRP. In the MSEERP, when $t$ is greater than $1100 \mathrm{~s}$, packet number received by sink gradually decreases with increasing invalid nodes, while in the MREEMRP there are no invalid nodes, so that the received packet number by the sink gradually becomes more than that of the MSEERP after $1100 \mathrm{~s}$. When $t$ is equal to $1759 \mathrm{~s}$, almost all nodes are dead in the MSEERP, and there is no data sent to the sink. Whereas in the MREEMRP, the received packet number by the sink begins to decrease since 2500 $\mathrm{s}$. When $t$ is equal to $1759 \mathrm{~s}$, sensor nodes no longer send data to the sink. To sum up, the number of total data packets received by the sink in the MSEERP is about 229763, while that in the MREEMRP is about 368222.

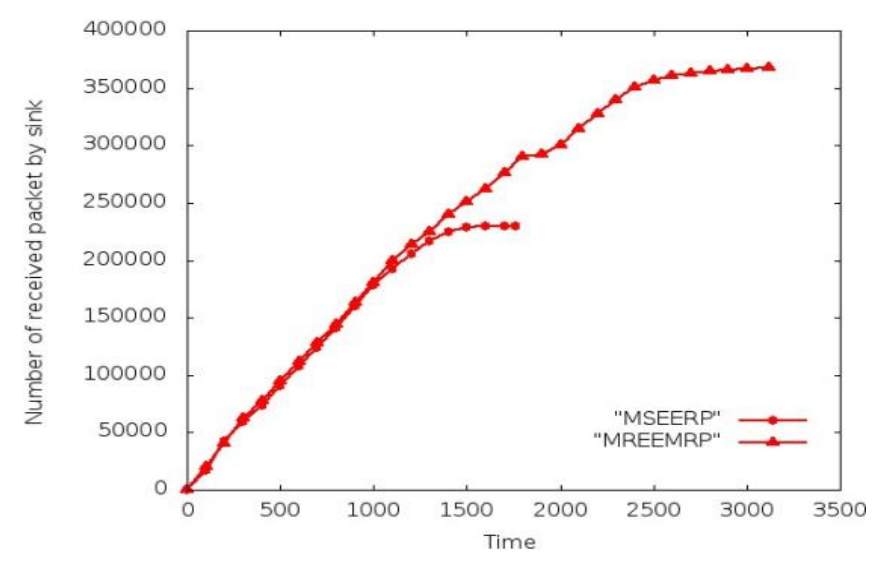

Figure 9. Comparison of Data Packet Received by Sink Node 


\section{Conclusions}

To overcome the disadvantages of high energy consumption and energy consumption imbalance in routing protocols with mobile sink of wireless sensor network, this paper proposes an energy-efficient mobile routing algorithm with mobile relays and sink. Based on the idea of grid, the MREEMRP selects the cluster head according to the residual energy of node and weighted sum of the distance between the node and the areal coordinates, which ensures the least energy consumption of data transmission, and avoids the node with less residual energy to be selected as the cluster head. When the mobile sink moves to the cluster head through controllable moving strategy, the cluster head can transmit data to the sink. Moreover, relay node with unlimited energy is introduced to take forward all packets from an ordinary node when it moves to the position of the ordinary node, and the node only needs to send its own sensing data.

Finally, the MREEMRP is simulated by the NS2 from three aspects of total energy consumption, active node number and received packet by sink, and comparison analysis with the MSEERP algorithm is also carried out. The results show that the MREEMRP is better than the MSEERP in all the three aspects, and that the MREEMRP can effectively reduce node forwarding data and energy consumption, thus extending the lifetime of nodes and the network.

\section{References}

[1] J. Zheng and B. X. Zhang, 'Wireless Sensor Network Technology", Beijing: China Machine Press, (2012).

[2] J. Luo and J. P. Huang, "Joint Mobility and Routing for Lifetime Elongation in Wireless Sensor Networks", Proceedings IEEE Infocom, (2005); Miami, FL, United states.

[3] E. M. Royer, P. M. M. Smith and L. E. Moser, "An analysis of the optimum node density for Ad hoc mobile networks", Proceedings of International Conference on Communications, (2001); Helsinki, Finland.

[4] K. Tian, B. Zhang, K. Huang and J. Ma, "Data Gathering Protocols for Wireless Sensor Networks with Mobile Sinks", Proceedings IEEE Global Communications Conference, (2010); Miami, FL, USA.

[5] H. Luo, F. Ye, J. Cheng, S. Lu and L. Zhang, "TTDD: Two-tier Data Dissemination in Large-scale Wireless Sensor Networks", Wireless Networks, vol. 161, no. 175, (2005).

[6] Y. Bi, L. Sun, J. Ma, I. Khan and C. Chen, "HUMS: An Autonomous Moving strategy for Mobile Sinks in Data-Gathering Sensor Network", Eurasip Journal on Wireless Communications and Networking, (2007).

[7] I. Chatzigiannakis, A. Kinalis and S. Nikoletseas, "Efficient data propagation strategies in wireless sensor networks using a single mobile sink", Computer Communications, vol. 896, no. 914, (2008).

[8] A. K. Chatzigiannakis, S. Nikoletseas and J. R. Rolim, "Fast and Energy Efficient Sensor Data Collection by Multiple Mobile Sinks", Proceedings of the 5th ACM International Workshop on Mobility Management and Wireless Access, (2007); Chania, Crete Island, Greece.

[9] N. M. Khan, I. Ali, Z. Khalid, G. Ahmed, A. A. Kavokin and R. Ramer, "Quasi centralized clustering approach for energy-efficient and vulnerability-aware routing in wireless sensor networks", Proceedings of the International Symposium on Mobile Ad Hoc Networking and Computing, (2008) ; Hong Kong, China.

[10] W. Wang, V. Srinivasan and K. C. Chua, "Extending the Lifetime of Wireless Sensor Networks Through Mobile Relays", IEEE/ACM Transactions on Networking, vol. 1108, no. 1120, (2008).

[11] Y. Xu, J. Heidemann and D. Estrin, "Geography-informed energy conservation for ad hoc routing", Proceedings of the Annual International Conference on Mobile Computing and Networking, (2001); Rome, Italy.

[12] Z. G. Lin, H. Q. Zhang, X. Y. Wang, F. Q. Yao and Z. X. Chen, "Energy-efficient Routing Protocol on Mobile Sink in Wireless Sensor Network", Advanced Materials Research, (2013); Guangzhou, China. 


\begin{abstract}
Authors
Lin Zhigui, he was born in 1974, Ph.D. He is an Associate Professor at school of Electronic and Information Engineering in Tianjin Polytechnic University. His research interests are Underwater acoustic sensor networks, Design and Application of Embedded System and Intelligent Information Processing. He has published more than 50 research papers in journals and 3 books.
\end{abstract}
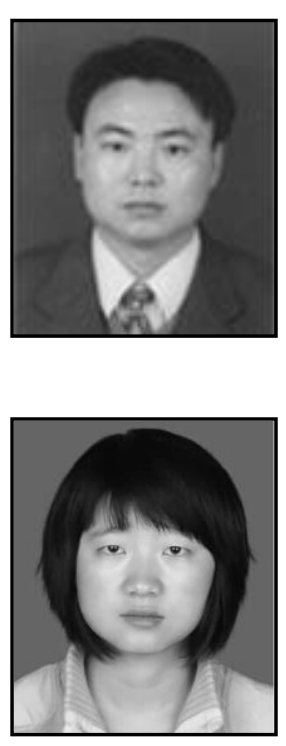

Li Min, she is currently a student at School of Electronic and Information Engineering in Tianjin Polytechnic University. Her research interests are Wireless sensor network, and Design and Application of Embedded System. 